\title{
Evolution of aptamers with a new specificity and new secondary structures from an ATP aptamer
}

\author{
ZHEN HUANG ${ }^{1}$ and JACK W. SZOSTAK ${ }^{2}$ \\ ${ }^{1}$ Department of Chemistry, Brooklyn College, Ph.D. Programs of Chemistry and Biochemistry, The Graduate School of CUNY, \\ Brooklyn, New York 11210, USA \\ ${ }^{2}$ Howard Hughes Medical Institute, Department of Molecular Biology, Massachusetts General Hospital, Boston, Massachusetts 02114, USA
}

\begin{abstract}
Small changes in target specificity can sometimes be achieved, without changing aptamer structure, through mutation of a few bases. Larger changes in target geometry or chemistry may require more radical changes in an aptamer. In the latter case, it is unknown whether structural and functional solutions can still be found in the region of sequence space close to the original aptamer. To investigate these questions, we designed an in vitro selection experiment aimed at evolving specificity of an ATP aptamer. The ATP aptamer makes contacts with both the nucleobase and the sugar. We used an affinity matrix in which GTP was immobilized through the sugar, thus requiring extensive changes in or loss of sugar contact, as well as changes in recognition of the nucleobase. After just five rounds of selection, the pool was dominated by new aptamers falling into three major classes, each with secondary structures distinct from that of the ATP aptamer. The average sequence identity between the original aptamer and new aptamers is $\mathbf{7 6} \%$. Most of the mutations appear to play roles either in disrupting the original secondary structure or in forming the new secondary structure or the new recognition loops. Our results show that there are novel structures that recognize a significantly different ligand in the region of sequence space close to the ATP aptamer. These examples of the emergence of novel functions and structures from an RNA molecule with a defined specificity and fold provide a new perspective on the evolutionary flexibility and adaptability of RNA.
\end{abstract}

Keywords: Aptamer; specificity; fold; selection; RNA evolution

\section{INTRODUCTION}

Nucleic acids can fold into a variety of three-dimensional shapes with specific structural, ligand-binding, and catalytic properties (for reviews, see Feigon et al. 1996; Lorsch and Szostak 1996; Norris et al. 2000; Mandal et al. 2003). Considering that RNAs contain only four rather similar building blocks (A, C, G, and U), the structural diversity of RNA is surprisingly large. RNAs can respond to selection pressure by evolving from one function to a different one through changes in sequence and fold (for reviews, see Famulok 1999; Wilson and Szostak 1999). As functional RNAs and DNAs have potential as catalysts for organic synthesis ( $\mathrm{Li}$ et al. 2000; Santoro et al. 2000), modules for ribozyme biosensors (Brody and Gold 2000; Jhaveri et al. 2000), therapeutics (Sioud 1999; Sioud and Leirdal 2000; Mayer et al. 2001), and tools in molecular biology (Gold et al. 1997;

Reprint requests to: Zhen Huang, Department of Chemistry, Brooklyn College, Ph.D. Programs of Chemistry and Biochemistry, The Graduate School of CUNY, Brooklyn, NY 11210, USA; e-mail: zhuang@brooklyn. cuny.edu; fax: (718) 951-4607.

Article and publication are at http://www.rnajournal.org/cgi/doi/ 10.1261/rna.5990203.
Gebhardt et al. 2000), understanding the relationship between specificity and structure (Tarasow et al. 2000) will aid in the design and evolution of catalysts and aptamers, as well as helping us to understand RNA evolution in nature. Attempts to evolve RNAs with altered specificity using in vitro directed evolution have been made previously (for reviews, see Famulok 1999; Wilson and Szostak 1999). For example, arginine aptamers have been evolved from a citrulline aptamer after 30\% mutagenesis (Famulok 1994). Half of the isolated aptamers retained the original structural framework and secondary structure while displaying the novel arginine binding specificity. The switch in specificity for the amino acid was achieved by only three mutations out of 44 nucleotides (Yang et al. 1996). Evolution of GTPbinding sites demonstrated that even a single nucleotide substitution within the same structural framework can lead to dual arginine and GTP binding specificity (Connell and Yarus 1994). An RNA aptamer binding to both guanine and xanthine was isolated from a random pool (Kiga et al. 1998), and naturally-occurring guanine-recognizing mRNA motifs functioning as riboswitches have also been identified in vivo (Mandal et al. 2003). There is no sequence homology between the unnaturally- and naturally-occurring 
aptamers. The naturally-occurring aptamers bind to guanine at lower nanomolar levels. Although guanine binds tightly to the dual guanine/xanthine aptamer $\left(\mathrm{K}_{\mathrm{d}}, 1.8 \mu \mathrm{M}\right)$, guanosine binds to this aptamer with $\sim 100$-fold less affinity, suggesting unfavorable interactions between the ribose and the aptamer structure.

Evolving a desired specificity while retaining the original structural framework requires minimizing the extent of mutagenesis. However, retention of the initial fold could limit the discovery of novel specificities, which might only be achievable in some cases in the context of a new fold. We were interested in addressing this issue, as part of a broader attempt to understand the evolutionary adaptability and flexibility of RNA.

A specific ATP-binding RNA motif (the ATP aptamer) was previously isolated by in vitro selection from a pool of random RNA sequences (Sassanfar and Szostak, 1993). The recognition loop sequences of the ATP aptamer (Fig. 1A) are the purine-rich 11-nucleotide loop-1 and the single-base loop-2 (the bulged G), and they are flanked by duplexes that serve to anchor and orient their ends. The solution structure of the ATP aptamer, determined independently by two laboratories, has revealed that the recognition loop forms a compact $\zeta$ (zeta)-shaped structure, with the ATP stacked at the end of the consecutive GAA to form a structure similar to a stable GNRA tetraloop (Dieckmann et al. 1996; Jiang et al. 1996). The ATP ribose sugar makes contacts with the aptamer, whereas the triphosphate points away from the aptamer and contacts solvent molecules. Attempts to convert the ATP aptamer to aptamers binding different ligands, such as GTP, by rational design based on the structure have so far failed. To evolve aptamers with a new specificity and structure, we selected aptamers for binding to a ligand with multiple changes from ATP in the nucleobase and sugar moieties (Fig. 1B,C). We used a 15\%-mutagenized pool to allow for the exploration of a broad region of structural space, while remaining in the region of sequence space close to the original aptamer. Here we describe novel aptamers with altered specificity and secondary structure derived from the mutagenized ATP aptamer pool.

\section{RESULTS}

The selection started from a library of $10^{14}$ mutagenized ATP-binding RNA se- quences (Fig. 1D). Random mutagenesis at 15\% per positions was programmed during the solid-phase synthesis of the DNA version of the library. The library was designed to contain a central region based on the ATP aptamer (40 nucleotides in length), as well as two flanking constant sequences (20 nucleotides in length) that serve as primer binding sites for reverse transcription, polymerase chain reaction (PCR), and transcription. Isolation of RNAs with a modified specificity was done by affinity chromatography on a modified GTP-agarose. The attachment chemistry transforms the ribose sugar to a substituted morpholine. We refer to the modified GTP as a morpholine-based GTP analog, or MBGT, and the resin as MBGT-agarose. To detect RNAs and collect appropriate fractions, RNAs were internally labeled with $\alpha-{ }^{32} \mathrm{P}-\mathrm{UTP}$ during transcription. Although we did not use a precolumn for this selection, nonspecific matrix-binding RNAs were not encountered. More than $99 \%$ of the nonspecifically binding RNAs are removed after a six-column-volume wash with binding buffer. Specifically binding RNAs were eluted with binding buffer con-

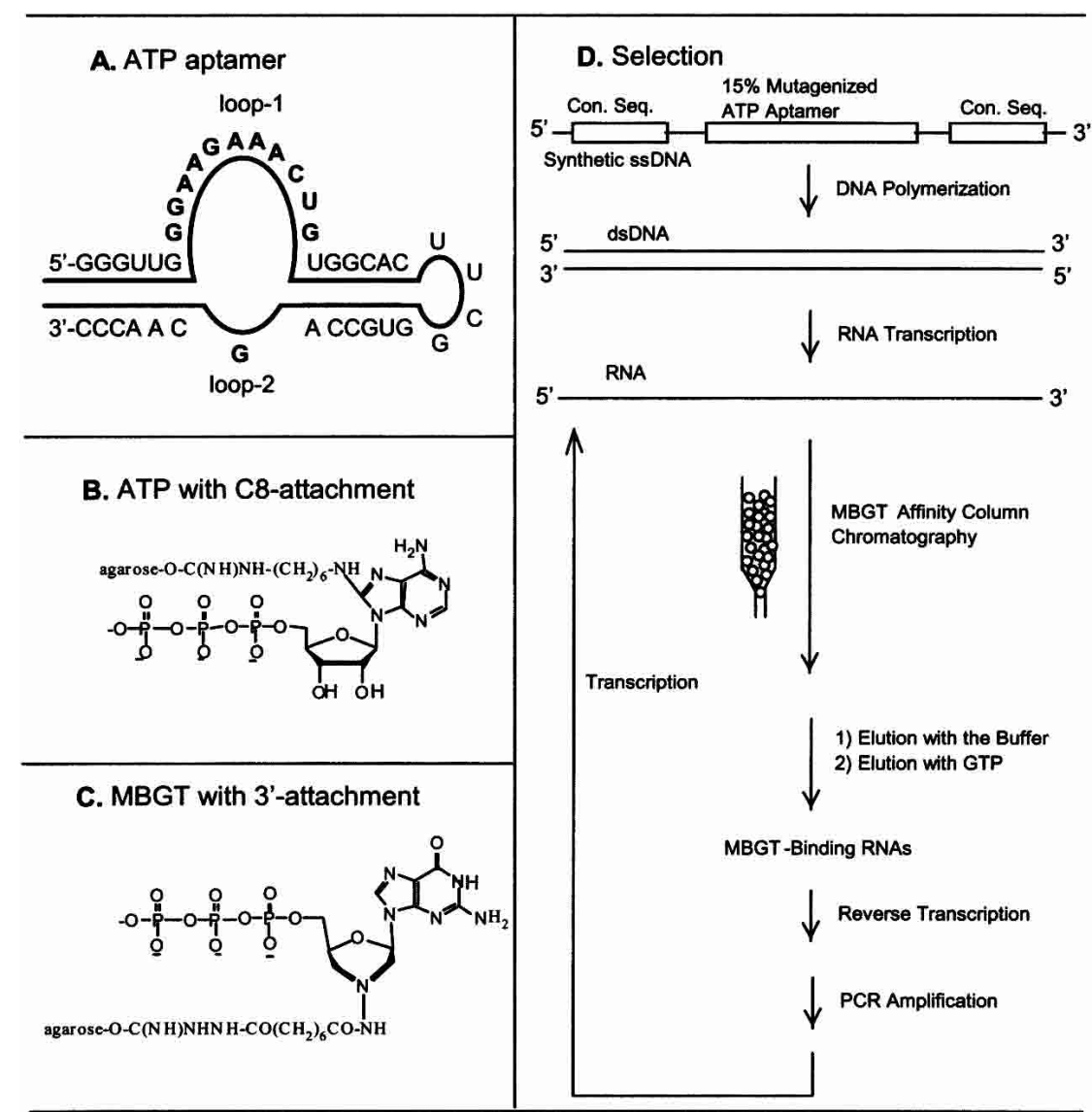

FIGURE 1. Selection scheme used to evolve the ATP aptamer to MBGT/GTP aptamers. $(A)$ ATP aptamer secondary structure. (B) ATP attached via C8 to agarose. (C) MBGT attached via the morpholine-N to agarose. $(D)$ The initial DNA library contained a 40-nucleotide ATP-aptamer region mutagenized at $15 \%$ per position, flanked by constant sequence regions. Transcribed RNA was applied to the MBGT affinity resin for aptamer selection. Bound RNA eluted with GTP was amplified by reverse transcription-PCR, and the resulting DNA was transcribed to yield RNA for the next round of selection. 
taining $4 \mathrm{mM}$ GTP. The selected RNAs therefore have a dual MBGT/GTP binding specificity. Given the differences between the ribose and morpholine moieties, this selective protocol is likely to focus aptamer-ligand interactions on the common guanine and triphosphate portions of the ligands.

MBGT/GTP aptamers were effectively enriched during the selection (Fig. 2). After the first, second, and third rounds of selection, only a small fraction of the input RNA was retained on the column after washing $(0.1 \%, 0.15 \%$, and $0.41 \%$ ). In the next two rounds of selection, the amount RNA retained on the column increase dramatically to $32 \%$ and $69 \%$, respectively, as MBGT/GTP binding RNAs came to dominate the population of RNA sequences. The retained RNA can be eluted with GTP, but not with ATP, CTP, or UTP. Approximately $10 \%$ of the input RNA remained on the column after the GTP elution in the fourth and fifth rounds of selection, and this material could be RNA that bound MBGT but not GTP. Only $0.1 \%$ of the RNA isolated from round 5 was retained by a morpholinebased adenosine triphosphate (MBAT) agarose column, and $0.47 \%$ by C8-linked ATP-agarose column, compared with $86 \%$ retention of the original ATP aptamer on the C8-linked ATP-agarose column. Insignificant retention of the RNAs on both ATP-agarose columns indicated that the isolated RNAs did not bind to the matrix, and the ligand specificity of the aptamers switched from ATP to MBGT/ GTP.

To preserve sequence and structural diversity, stringent selection conditions were avoided, and RNA from the fifth round was cloned and sequenced (Fig. 3). All 34 active

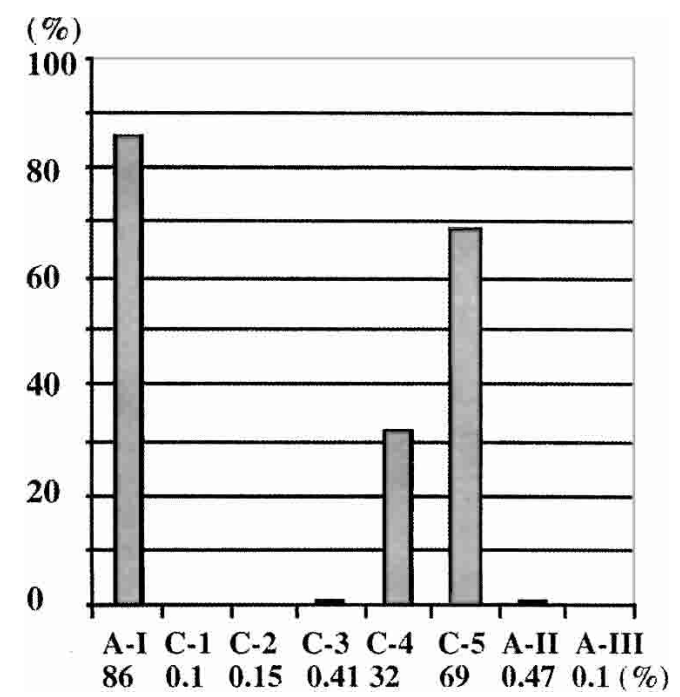

FIGURE 2. Retention of RNA on the ATP or MBGT column after six-column-volume wash. A-I and A-II show the retention of the original ATP aptamer and the cycle-5 RNA on ATP-C8-agarose columns. C-1, C-2, C-3, C-4, and C-5 show retention of cycle- 1 to cycle-5 RNAs on the MBGT-agarose columns. A-III shows the retention of the cycle-5 RNA on an MBAT-agarose column. sequences derived from these clones were unique, indicating that the sequence diversity of the fifth-round RNA was still high. However, closer inspection revealed that many of these sequences shared sequence motifs that were presented in a common secondary structural context. 59\% of the sequences (20 of 34 sequences) fell into three classes by these criteria: class I (five sequences, $15 \%$ of the population), class II (four sequences, 12\%), and class III (11 sequences, 32\%). The most active of the class I, II, and III aptamers were clones N10, N4, and G1, respectively. The following studies focus on these aptamers. The other 14 sequences (41\%) did not share obvious sequence or structural similarity and were not investigated further.

Deletion analysis of N10, N4, and G1 aptamers defined the minimal sequences required for their function (Fig. 4AC). These truncation experiments indicated that the $5^{\prime}$ constant sequence was not required for binding in the class I and II aptamers, but complete removal of the 3 '-constant sequence caused loss of binding affinity. In contrast, complete deletion of the $5^{\prime}$-constant sequence of the class III aptamer abolished its binding, whereas the $3^{\prime}$-constant sequence could be completely deleted without affecting its affinity, although a further deletion of the eight-nucleotide tail at the $3^{\prime}$-end caused loss of binding.

After identifying the minimal sequences relevant to class I and II function, a variety of constructs were made with compensatory mutations in the proposed stems or with changes in the recognition loops. N10-C/A was constructed from the minimum sequence of the class I clone N10 by altering the $\mathrm{C}$ to $\mathrm{A}$ in CAACUCG loop (so as to agree with the class I consensus sequence AAACUCG), and N4-1 was constructed from the minimum sequence of the class II clone N4 after repairing its mismatches. As expected, both N10-C/A (Fig. 4D) and N4-1 (Fig. 4E) bound to the MBGT matrix more effectively than their parental molecules. Both class I and II aptamers fold into secondary structures with two highly conserved loops flanked by base-paired stems. These two highly conserved loops are identical but are presented in opposite orientation (Fig. 4 D,E), strongly suggesting that these loops are the ligand-binding regions and that the stems serve simply to anchor the ends of the recognition loops. The secondary structures of the structurally equivalent class I and II aptamers were confirmed by making compensatory mutations in the stems, and were further confirmed by construction of a chimeric sequence (Fig. 4F) using loops 1I and 2I of class I to replace loops 1II and 2II of class II. This chimeric molecule (N10-N4) displayed the same level of affinity as the parental molecules, N10-C/A and N4-1.

The structure of the class III aptamer is more complex (Fig. 4G). Deletion experiments (Fig. 4C) and structure prediction using Mfold suggested that part of the $5^{\prime}$-constant sequence $\left(5^{\prime}\right.$-uguacgcc) of G1 (a class III aptamer) is involved in stem-1, and this was confirmed by analysis of compensatory mutations, and stem-2 was confirmed in the 


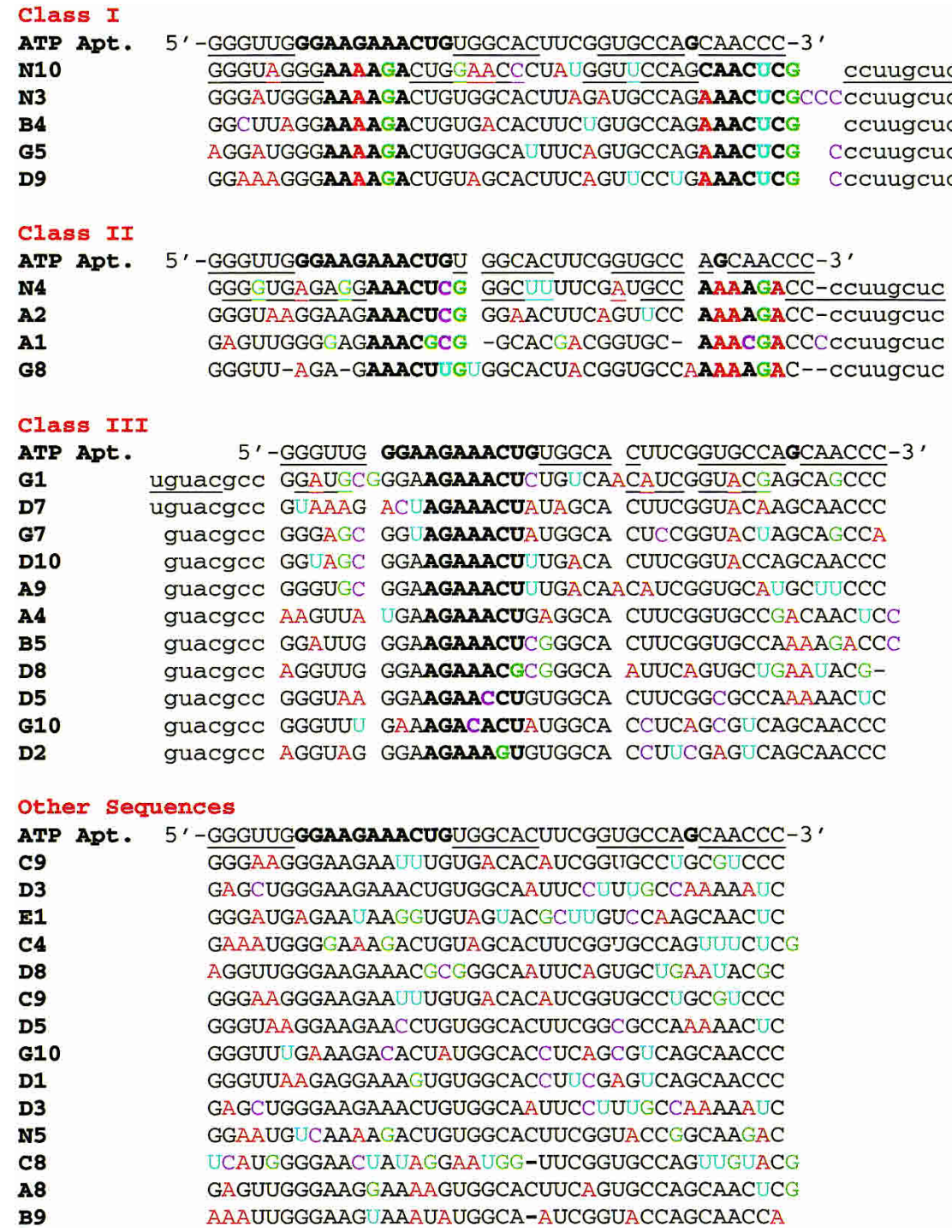

FIGURE 3. Sequences of the MBGT/GTP aptamers isolated after the fifth round of selection. Bold letters represent conserved recognition loop sequences. Mutated nucleotides are shown in color (change to A, red; to G, green; to U, blue; and to C, purple).

same way (Fig. 5). Deletion of the $3^{\prime}$-tail (3'-CCCGACGA) abolished the binding affinity, suggesting that this "tail" is essential for the binding, and removal of the conserved small loop also disrupted the binding.

The proposed secondary structures of the three classes were further confirmed by compensatory mutations (Fig. 5). For class I, three mutations on either strand of the external stem (mutants 1 or 2) weakened the stem, resulting in lower column binding (Fig. 5A). The compensatory double mutant $(1+2)$ restored both base pairing and column binding. Mutations on either strand of the internal stem (mutants 3 or 4 ) had a slightly stronger effect on weakening aptamer structural stability, as seen in the decreased column binding. As expected, compensatory mutations $(3+4)$ restored the stability and column binding ability of the class I aptamer. The secondary structures of the class II and class III aptamers were confirmed through similar compensatory mutation analysis (Fig. 5B,C). As mentioned before, deletion of the $3^{\prime}$-tail ( $3^{\prime}$ CCCGACGA) abolished binding.

Apparent $\mathrm{K}_{\mathrm{d}} \mathrm{s}$ of the class I, II, and III aptamers in solution (Fig. 5) were measured via equilibrium filtration (Jenison et al. 1994). The class I and II aptamers have apparent dissociation constants of $\sim 20 \mu \mathrm{M}$, while the class III aptamer $\mathrm{K}_{\mathrm{d}}$ is $\sim 33 \mu \mathrm{M}$. This moderate binding affinity is expected, as stringent conditions were purposely avoided during the selection. As pointed out previously, the class I and class II aptamers share the same binding loop in different orientations. The chimera (N10-N4), constructed using the binding loops of class I and the stems of class II, has the same ligand binding affinity as its parents (Fig. 5D), as expected.

\section{DISCUSSION}

We have used mutagenesis and in vitro selection to evolve an ATP aptamer into a series of new MBGT/GTP aptamers that retain $76 \%$ average sequence identity to the original ATP aptamer sequence. These new RNAs no longer bind to the original ligand (ATP), suggesting that the evolution of a new specificity is easier than the isolation of binders with dual specificity, which would have to rely on fewer sites of interaction between aptamer and ligand. In addition, the structure of the original ATP aptamer was completely altered by mutations in the three characterized classes of the selected MBGT/GTP aptamers. Remarkably, these aptamers arose independently many times, using different combinations of mutations to destroy the same original secondary structure and to build the new secondary structure. It appears that new secondary structures and new recognition loops are required to allow binding to the MBGT-derivatized matrix and elution with GTP. Probably because of the nonstringent selection pressure, a variety of sequences with moderate affinity $(20-33 \mu \mathrm{M})$ have been preserved in the final selected pool. The selected GTP/MBGT aptamers have completely different conserved sequences and secondary structures from the previously isolated guanine/xanthine aptamer (Kiga et al. 1998) and the naturally-occurring guanine aptamer (Mandal et al. 2003), indicating that similar binding functions can be performed by different sequences and folds. 


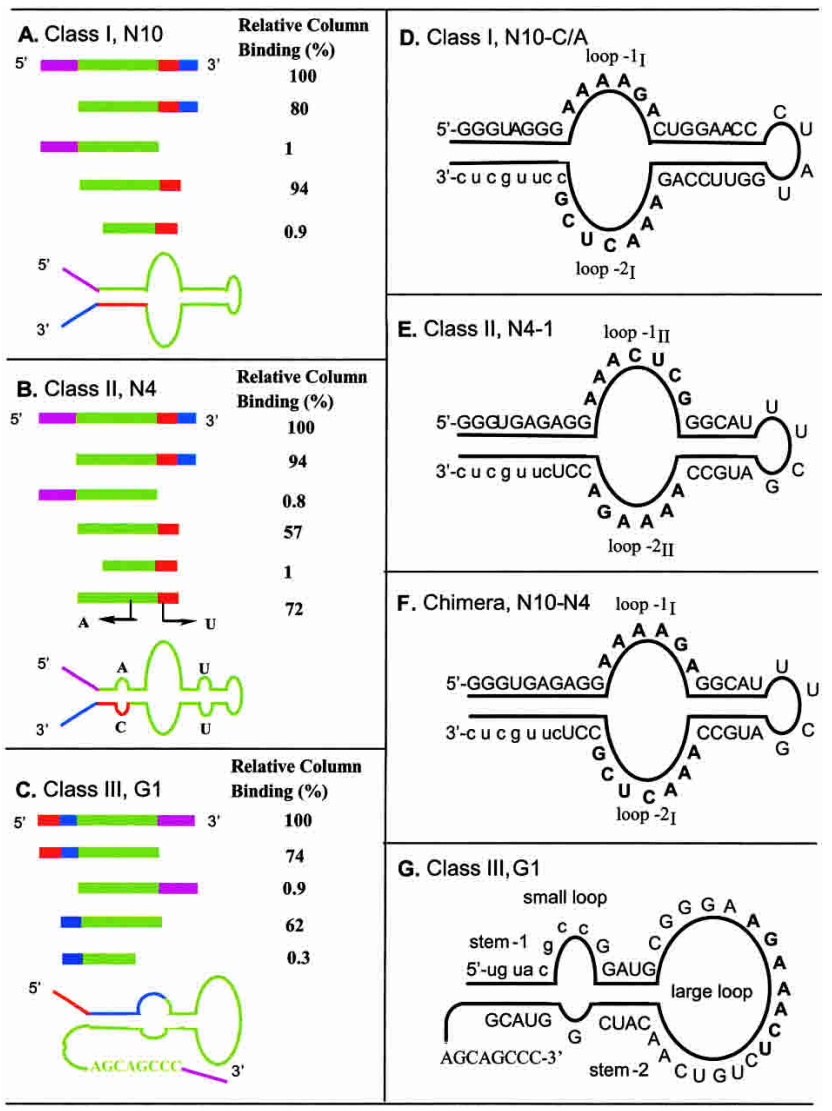

FIGURE 4. Deletion analysis and secondary structures of the class I, II, and III aptamers. The color coding of the aligned deletion constructs matches the secondary structure schematic shown below, and the full sequence of each aptamer is shown as a secondary structure diagram to the right.

It is interesting that many of the selected mutations were in the original duplexes, which are the most common and important structural element in RNA architecture (Fan et al. 1996). It appears that disruption of old structures is essential to allow efficient folding into a new structure. In the class I aptamer N10, both of the original stems of the ATP aptamer were disrupted and replaced by a different secondary structure (Fig. 6A,B). The original internal stem was eliminated, and a new internal stem was formed, as a result of mutations causing a shift in register of two nucleotides in the paired sequences (Fig. 6B). The original external stem was disrupted by mutations in its $3^{\prime}$-segment, which formed loop-2I (seven nucleotides). The $3^{\prime}$-constant sequence $\left(3^{\prime}\right.$-cucguucc) was recruited to form the new external stem. MBGT-agarose binding was increased after changing the $3 \mathrm{U} / \mathrm{G}$ wobble pairs in the new external stem to Watson-Crick C/G pairs. Part of the original loop-1, altered by two mutations, became loop-1I (six nucleotides). The new loop-1I and loop-2I are highly conserved among class I aptamers, suggesting that they are largely responsible for the new ligand specificity of the aptamer.

The class II aptamer N4 retained the original internal tetraloop, and largely maintained its internal stem through compensatory mutations (Fig. 6C,D), although the original base pair next to the bulged $\mathrm{G}$ was disrupted. Loop-1II was formed from part of the original loop- 1 of the ATP aptamer. The original $3^{\prime}$-segment of the external stem was reconstructed by three mutations to become a part of loop2II. As in the class I aptamer N10, part of the $3^{\prime}$-constant sequence was recruited to form a new external stem. The mutations that create the class II aptamer specificity and secondary structure can therefore be classified into the same categories as those in class I, that is, disruption of the old secondary structure, creation of the new secondary structure, and creation of the new recognition loops. Similarly, many of the selected mutations in class III clone G1 served to disrupt the old stems and cause a large secondary structure rearrangement (Fig. 6E,F). Although some mutations serve more than one function, mutations in the original duplexes may be critical for the evolution of new structures.

An RNA library based on the ATP aptamer but mutagenized at a lower rate $(6 \%)$ has so far failed to yield MBGT/GTP aptamers. Although selections can fail for many unrelated reasons, it is possible that sequences containing enough mutations to simultaneously alter both

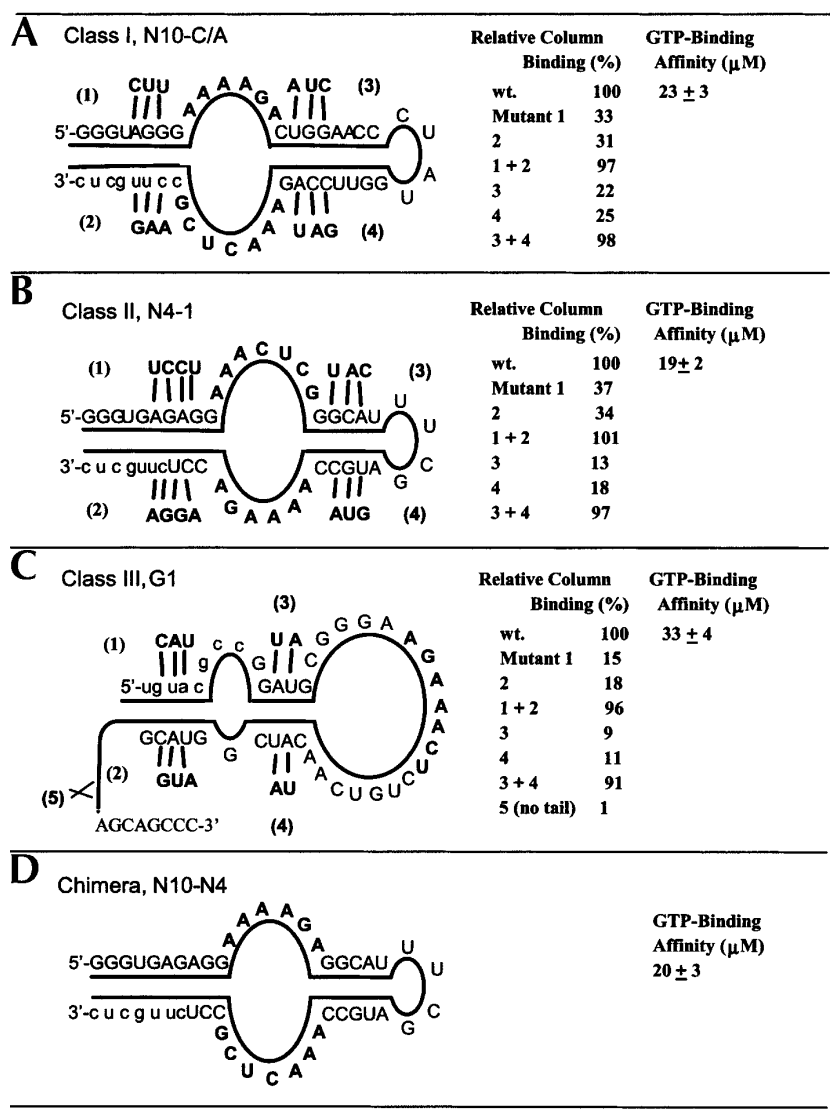

FIGURE 5. Compensatory mutations, secondary structures, and binding affinities of the class I, II, and III aptamers. Column binding was determined after six-column-volume wash using the binding buffer. 
6A. N10 (Class I) evolved from the ATP Aptamer

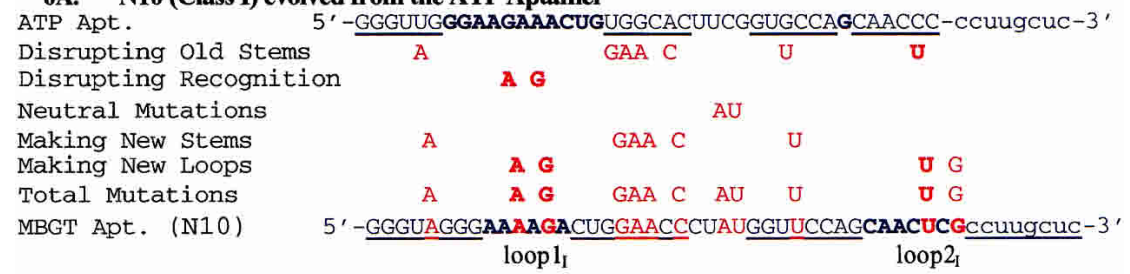

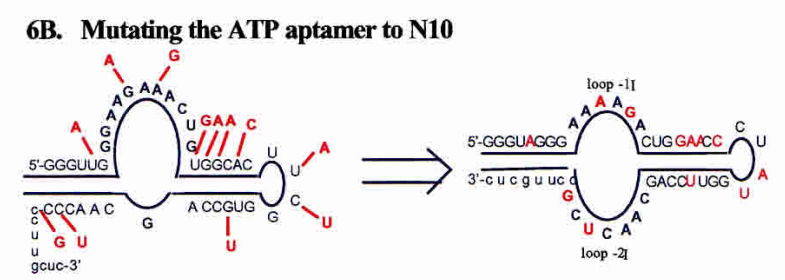

6C. N4 (Class II) evolved from the ATP Aptamer

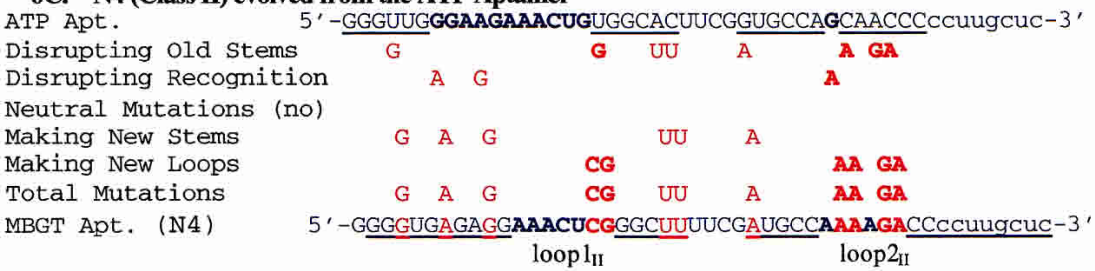

6D. Mutating the ATP aptamer to N4

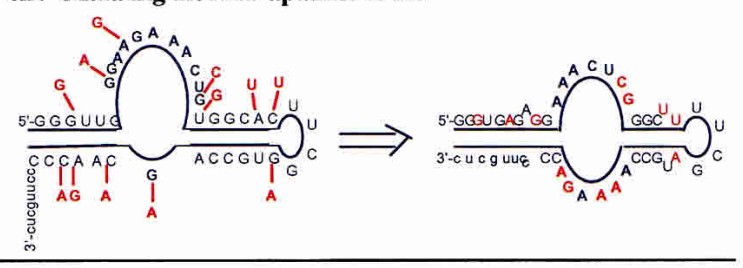

6E. G1 (Class III) evolved from the ATP Aptamer

ATP Apt. 5'-uguacgCCGGGUUG GGAAGAAACUGUGGCA CUUCGGUGCCAGCAACCC-3' Disrupting old Stems Disrupting Recognition Neutral Mutations Making New Stems Making New Loops Total Mutations MBGT Apt. (G1)
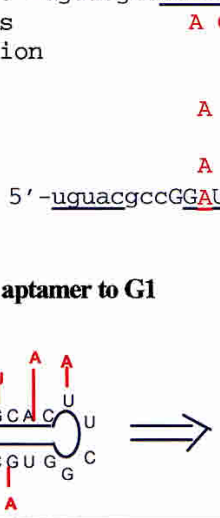
A GC G $A G^{G}$ A GCG (a) $\mathrm{C}^{\mathrm{UAA}} \mathrm{A}$ C U A A A G G Large loop

\section{F. Mutating the ATP aptamer to G1}

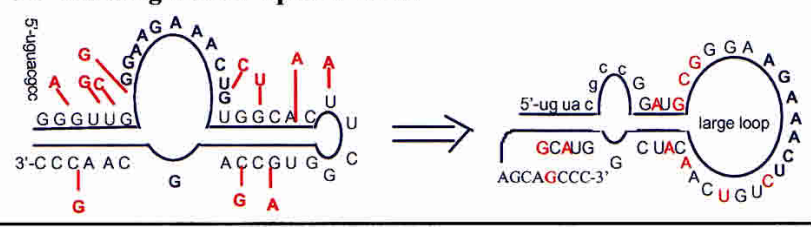

FIGURE 6. Interpretation of mutations leading to class I, II, and III aptamers. Bold nucleotides are in highly conserved recognition loops, and mutations are highlighted in color. $(A, C$, $E$ ) Original ATP aptamer sequence aligned with evolved MBGT aptamer sequence. Mutations with different potential roles are shown in between the initial and final sequences. $(B, D, F)$ The same mutations are illustrated on secondary structure sequence diagrams to illustrate the location and roles of the selected mutations. specificity and fold are extremely rare in this library. Indeed, the average mutation rate of the MBGT/GTP aptamers (24\%) was significantly higher than the mutation rate $(15 \%)$ of the initial ATP aptamer library. Our results are consistent with predictions of RNA secondarystructure evolution, in which $\sim 20 \%$ mutation is generally required to switch a random RNA sequence from one secondary structure to another (Huynen 1996). Our results also indicate that a specific binding function can be achieved by several different structures, showing not only that one function may have multiple structural solutions in RNA sequence space but also that distinct structures may lie relatively close in sequence space to an arbitrary starting point. Computational simulation of RNA evolutionary adaptation suggests that extensive neutral networks connect structural and functional neighbors (Schuster et al. 1994; Fontana and Schuster 1998). It has been recently demonstrated that two completely unrelated ribozymes with different folds and catalytic functions can be evolved from a single RNA via a series of neutral mutations (Schultes and Bartel 2000). Our experimental observations provide another example to suggest that RNA structures may tend to evolve through the accumulation of mutations followed by jumps to distinct structures with novel functions.

\section{MATERIALS AND METHODS}

\section{General molecular biology techniques}

In vitro transcription reactions were conducted in $26 \mathrm{mM} \mathrm{MgCl} ; 40 \mathrm{mM}$ Tris $(\mathrm{pH}$ 7.9); $2.5 \mathrm{mM}$ spermidine; $0.01 \%$ Triton $\mathrm{X}-100 ; 5 \mathrm{mM}$ of each ATP, CTP, and UTP; 9 mM GTP; $10 \mathrm{mM} \mathrm{DTT;} 200 \mathrm{U} / \mathrm{mL}$ RNAsin (Promega, $40 \mathrm{U} / \mu \mathrm{L}$ ); $2000 \mathrm{U} / \mathrm{mL}$ T7 RNA polymerase (New England Biolabs); and 0.1$0.5 \mu \mathrm{M}$ template and were incubated for $10 \mathrm{~h}$ at $37^{\circ} \mathrm{C}$. Reverse transcription reactions were conducted in $10 \mathrm{mM}$ DTT, $1 \mathrm{mM}$ of each dNTP, $0.1-1 \mu \mathrm{M}$ DNA primer, RNA template up to $0.2 \mu \mathrm{M}$, and $10 \mathrm{U} / \mu \mathrm{L}$ reverse transcriptase (superscript II and $5 \times$ buffer; GIBCO BRL). After incubation for $2 \mathrm{~h}$ at $42^{\circ} \mathrm{C}, 5 \mathrm{M}$ $\mathrm{NaOH}$ was added to a final concentration 
of $100 \mathrm{mM}$. This solution was heated for $10 \mathrm{~min}$ at $90^{\circ} \mathrm{C}$ to digest the RNA template, followed by neutralization and $\mathrm{EtOH}$ precipitation of the cDNA. PCR reactions were conducted in $50 \mathrm{mM} \mathrm{KCl}$, $10 \mathrm{mM}$ Tris (pH 8.5), 0.1\% Triton X-100, $0.2 \mathrm{mM}$ of each dNTP, $2.5 \mathrm{mM} \mathrm{MgCl}_{2}, 1 \mu \mathrm{M}$ of each primer, and $50 \mathrm{U} / \mathrm{mL}$ Taq DNA polymerase. Thermocycling was between $94^{\circ} \mathrm{C}(45 \mathrm{sec}), 72^{\circ} \mathrm{C}(2$ $\min )$, and $50^{\circ} \mathrm{C}(1 \mathrm{~min}$ and $45 \mathrm{sec})$.

\section{Cloning and sequencing}

TOPO PCR cloning kit (Invitrogen) was used to clone the PCR products, and plasmids were isolated from transformed cells and sequenced by the dideoxy method.

\section{Truncation and site-directed mutation}

RNAs containing these mutations were prepared by transcription of synthetic DNA templates made by solid-phase synthesis.

\section{RNA pool construction}

The library was chemically synthesized as a DNA oligonucleotide (5'-AGCCAGCTTAATGAGCAAGGgggttgctggcaccgaagtgccacagttt cttcccaaccc-GGCGTACATGCAACCACTCC-TATAGTGAGTCG TATTACGC- $3^{\prime}$, where the lowercase letters indicate the $15 \% \mathrm{mu}$ tagenized ATP aptamer region, uppercase sequences are the flanking constant sequences, and the underlined sequence is the T7 RNA polymerase promoter). The synthetic DNA was purified by polyacrylamide gel electrophoresis (PAGE). The quality of the synthesized DNA (Fig. 1D) was assessed by a primer extension reaction using 20-fold molar excess of DNA template and ${ }^{32} \mathrm{P}$-labeled 3 '-primer. This experiment indicated that $18 \%$ of the DNA could be copied into a full-length complementary strand. Based on 1 nmole of purified synthetic DNA $\left(6 \times 10^{14}\right.$ molecules $)$ and the fraction of amplifiable sequences, we estimate that the complexity of the DNA library used to begin the selection was $\sim 1 \times 10^{14}$ independent molecules. Twenty clones from the DNA library were sequenced to confirm the mutagenic rate and random distribution of mutations in the aptamer region. The RNA pool was prepared from the DNA library by T7 RNA polymerase transcription in the presence of $\left[\alpha-{ }^{32} \mathrm{P}\right]-\mathrm{GTP}$ or $\left[\alpha-{ }^{32} \mathrm{P}\right]-\mathrm{UTP}$. The transcribed RNA was purified by PAGE, and each extendable DNA template yielded 40 RNA copies on average.

\section{Affinity selection procedures}

A column of MBGT-agarose beads (0.4-1.5 mL, $2.2 \mathrm{mM}$ MBGT immobilized to the matrix via an adipic acid dihydrazide linker; Sigma-Aldrich) was pre-equilibrated with the binding buffer (10$30 \mathrm{~mL}, 300 \mathrm{mM} \mathrm{NaCl}, 5 \mathrm{mM} \mathrm{MgCl}, 20 \mathrm{mM}$ Tris- $\mathrm{HCl}$ at $\mathrm{pH}$ 7.6). Transcribed RNA $(100 \mu \mathrm{L}, 0.1-2 \mathrm{mM})$ was heated for $1 \mathrm{~min}$ at $65^{\circ} \mathrm{C}$ and allowed to gradually cool to room temperature, followed by addition of $10 \times$ binding buffer $(11 \mu \mathrm{L})$. This solution was allowed to stand for $15 \mathrm{~min}$ at room temperature. The RNA solution was loaded on the MBGT-agarose column, the column was washed with binding buffer (six column-volumes), and one-column-volume fractions were collected. The remaining RNA was eluted with three column-volumes of 4 mM GTP (MBGT analog) in binding buffer. A scintillation counter was used to determine the radioactivity in the fractions and that remaining on the aga- rose. The fractions from the GTP elution were combined and precipitated in ethanol, and the residual salt was removed by G-25 Sepharose gel filtration. The isolated RNA was reverse transcribed, the cDNA was amplified by PCR, and the amplified DNA was then transcribed into RNA again for the next cycle of selection.

\section{Binding assays}

MBGT-agarose binding experiments were performed with PAGEpurified RNA (2-40 pmole). The RNAs were loaded onto $0.4 \mathrm{~mL}$ bed-volume MBGT agarose columns, which were pre-equilibrated with binding buffer. After incubation at room temperature, columns were eluted with binding buffer (six column-volumes), followed by elution with three column-volumes of $1 \times$ binding buffer containing $4 \mathrm{mM}$ GTP. Fractions of half-column volume were collected, and radioactivity in each fraction was quantitated by a scintillation counting.

Dissociation constants in solution were determined by equilibrium filtration (Jenison et al. 1994). Using this technique, the bound and unbound ligand (GTP) partition between a membrane. The solution $(200 \mu \mathrm{L})$ of the ligand $\left(0.5 \mathrm{nM}, \alpha-{ }^{32} \mathrm{P}-\mathrm{GTP}\right)$ and the aptamer $(0.5-200 \mu \mathrm{M})$ in the binding buffer were incubated for 15 min at $25^{\circ} \mathrm{C}$ prior to loading in Mirocon YM-30 unit. The solution was centrifuged at $13,000 \mathrm{~g}$ for $10 \mathrm{sec}$ to saturate the membrane, and the filtrate was transferred back to the unit. The solution was centrifuged for another $20 \mathrm{sec}$, and the filtrate $(-25 \mu \mathrm{L})$ was collected. Aliquots $(20 \mu \mathrm{L})$ were taken from both the remaining solution and the filtrate, and radioactivity in each aliquot was quantitated by a scintillation counting. GTP passed almost freely through the membrane in this protocol, and the aptamers were nearly completely prevented from moving through the membrane under these conditions. The concentration of the aptamer-bound ligand was calculated from the difference between the ligand in the filtrate and in the remaining solution. Curves of the bound-ligand fractions versus the aptamer concentrations were made, and the apparent $\mathrm{K}_{\mathrm{d}} \mathrm{s}$ were calculated from $50 \%$ of the ligand binding.

\section{ACKNOWLEDGMENTS}

We thank Jonathan Davis, John Marino, David Wilson, Kourosh Salehi-Ashtiani, and James Godde for comments on the manuscript, as well as the members of our laboratories for discussions and help. Z.H. was supported by PSC-CUNY Research Awards and CUNY Project Programs (\#63193-00-32, \#64203-00-33, \#65356-0034, \#80210-0302, and \#80209-0408), and J.W.S. was supported by NIH grant GM 53936. J.W.S. is an Investigator of the Howard Hughes Medical Institute.

The publication costs of this article were defrayed in part by payment of page charges. This article must therefore be hereby marked "advertisement" in accordance with 18 USC section 1734 solely to indicate this fact.

Received June 2, 2003; accepted August 18, 2003.

\section{REFERENCES}

Brody, E.N. and Gold, L. 2000. Aptamers as therapeutic and diagnostic agents. J. Biotechnol. 74: 5-13.

Connell, G.J. and Yarus, M. 1994. RNAs with dual specificity and dual 
RNAs with similar specificity. Science 264: 1137-1141.

Dieckmann, T., Suzuki, E., Nakamura, G.K., and Feigon, J. 1996. Solution structure of an ATP-binding RNA aptamer reveals a novel fold. RNA 2: 628-640.

Famulok, M. 1994. Molecular recognition of amino acids by RNAaptamers: An L-citrullin binding RNA motif and its evolution into an L-arginine binder. J. Am. Chem. Soc. 116: 1698-1706.

. 1999. Oligonucleotide aptamers that recognize small molecules. Curr. Opin. Struct. Biol. 9: 324-329.

Fan, P., Suri, A.K., Fiala, R., Live, D., and Patel, D.J. 1996. Molecular recognition in the FMN-RNA aptamer complex. J. Mol. Biol. 258: $480-500$.

Feigon, J., Dieckmann, T., and Smith, F.W. 1996. Aptamer structures from A to $\zeta$. Chem. Biol. 3: 611-617.

Fontana, W. and Schuster, P. 1998. Continuity in evolution: On the nature of transitions. Science 280: 1451-1455.

Gebhardt, K., Shokraei, A., Babaie, E., and Lindqvist, B.H. 2000. RNA aptamers to S-adenosylhomocysteine: Kinetic properties, divalent cation dependency, and comparison with anti-S-adenosylhomocysteine antibody. Biochemistry 39: 7255-7265.

Gold, L., Brown, D., He, Y., Shtatland, T., Singer, B.S., and Wu, Y. 1997. From oligonucleotide shapes to genomic SELEX: Novel biological regulatory loops. Proc. Natl. Acad. Sci. 94: 59-64.

Huynen, MA. 1996. Exploring phenotype space through neutral evolution. J. Mol. Evol. 43: 165-169.

Jenison, R.D., Gill, S.C., Pardi, A., and Polisky, B. 1994. High-resolution molecular discrimination by RNA. Science 263: 1425-1429.

Jhaveri, S.D., Kirby, R., Conrad, R., Maglott, E.J., Bowser, M., Kennedy, R.T., Glick, G., and Ellington, A.D.J. 2000. Designed signaling aptamers that transduce molecular recognition to changes in fluorescence intensity. J. Am. Chem. Soc. 122: 2469-2473.

Jiang, F., Kumar, R.A., Jones, R.A., and Patel, D.J. 1996. Structural basis of RNA folding and recognition in an AMP-RNA aptamer complex. Nature 382: 183-186.

Kiga, D., Futamura, Y., Sakamoto, K., and Yokoyama, S. 1998. An RNA aptamer to the xanthine/guanine base with a distinctive mode of purine recognition. Nucleic Acids Res. 26: 1755-1760.
Li, Y., Liu, Y., and Breaker, R.R. 2000. Capping DNA with DNA. Biochemistry 39: 3106-3114.

- 1996. Chance and necessity in the selection of nucleic acid catalysis. Acc. Chem. Res. 29: 103-110.

Mandal, M., Boese, B., Barrick, J.E., Winkler, W.C., and Breaker, R.R. 2003. Riboswitches control fundamental biochemical pathways in Bacillus subtilis and other bacteria. Cell 113: 577-586.

Mayer, G., Blind, M., Nagel, W., Bohm, T., Knorr, T., Jackson, C.L., Kolanus, W., and Famulok, M. 2001. Controlling small guaninenucleotide-exchange factor function through cytoplasmic RNA intramers. Proc. Natl. Acad. Sci. 98: 4961-4965.

Norris, J.S., Hoel, B., Voeks, D., Maggouta, F., Dahm, M., Pan, W., and Clawson, G. 2000. In vitro selection of functional nucleic acids. Adv. Exp. Med. Biol. 465: 293-301.

Santoro, S.W., Joyce, G.F., Sakthivel, K., Gramatikova, S., and Barbas III, C.F. 2000. RNA cleavage by a DNA enzyme with extended chemical functionality. J. Am. Chem. Soc. 122: 2433-2439.

Sassanfar, M. and Szostak, J.W. 1993. An RNA motif that binds ATP. Nature 364: 550-553.

Schultes, E.A. and Bartel, DP. 2000. One sequence, two ribozymes: Implications for the emergence of new ribozyme folds. Science 289: 448-452.

Schuster, P., Fontana, W., Stadler, P.F., and Hofacker, I.L. 1994. From sequences to shapes and back: A case study in RNA secondary structures. Proc. R. Soc. Lond. B 255: 279-284.

Sioud, M. 1999. Application of preformed hammerhead ribozymes in the gene of cancer. Int. J. Mol. Med. 3: 381-384.

Sioud, M. and Leirdal, M. 2000. Therapeutic RNA and DNA enzymes. Biochem. Pharmacol. 60: 1023-1026.

Tarasow, T.M., Tarasow, S.L., and Eaton, B.E. 2000. RNA Diels-Alderases: Relationships between unique sequences and catalytic function. J. Am. Chem. Soc. 122: 1015-1021.

Wilson, D.S. and Szostak, J.W. 1999. In vitro selection of functional nucleic acids. Annu. Rec. Biochem. 68: 611-647.

Yang, Y., Kochoyan, M., Burgstaller, P., Westhof, E., and Famulok, M. 1996. Structural basis of ligand discrimination by two related RNA aptamers resolved by NMR spectroscopy. Science 272: 1343-1347. 